\title{
Monitoria acadêmica em enfermagem em doenças infecciosas e parasitárias: um relato de experiência
}

\author{
Academic monitoring in nursing in infectious and parasitic diseases: an experience report \\ Monitoría académica en enfermería en enfermedades infecciosas y parasitarias: un relato \\ de experiencia
}

Carla Costa da Silva ${ }^{1 *}$, Rubia Rodrigues Neves ${ }^{1}$, Victória Karolina Santos Santana ${ }^{1}$, Larissa dos Santos Almeida ${ }^{1}$, Ruth Carolina Leão Costa ${ }^{1}$, Cláudia dos Santos Ozela ${ }^{1}$, Tatyellen Natasha da Costa Oliveira ${ }^{1}$, Antonia Margareth Moita Sá1, laci Proença Palmeira ${ }^{1}$, Larissa Lima Figueira Freire ${ }^{1}$, Adriana Lorena Sena de Lima ${ }^{1}$, Brenda Lima Filocreão1, Thayná Maria Andrade Silva ${ }^{1}$, Mayane Silva Lopes ${ }^{1}$, Mônica de Cássia Pinheiro Costa ${ }^{1}$.

\section{RESUMO}

Objetivo: Relatar a experiência no processo ensino-aprendizagem da monitoria acadêmica do componente curricular Enfermagem em Doenças Infecciosas e Parasitárias (DIP). Relato de experiência: Trata-se de um estudo descritivo, de abordagem qualitativa, do tipo relato de experiência, baseado na vivência da monitoria acadêmica do componente curricular de DIP na Universidade do Estado do Pará, durante o período de setembro a dezembro de 2018. O discente-monitor em DIP foi responsável por acompanhar o docente no campo teórico e prático. Os momentos teóricos aconteceram em sala de aula, verificando os conteúdos ministrados, na organização e planejamento de revisões e materiais de apoio para os acadêmicos. $O$ momento de prática foi subdivido em DIP na Atenção Básica e DIP hospitalar, no qual o discente-monitor, em conjunto com o docente, atuou na orientação e execução de atividades, o que lhe proporcionou integração em atividades docentes, superando os desafios do cotidiano profissional, oportunizando-lhe experiências de consolidação para a sua formação. Considerações finais: O Programa de Monitoria amplia espaços de aprendizagem e contribui para uma formação profissional crítica, reflexiva e alinhada com o magistério superior.

Palavras-chave: Monitoria, Ensino, Aprendizagem.

\begin{abstract}
Objective: To report an experience in teaching-learning process of academic monitoring on nursing's curricular component in infectious and parasitic diseases (IPD). Experience report: this is a descriptive study, of qualitative approach, of experience's report type, based on the experience from the academic monitoring of curricular component of IPD in State University of Pará, from September to December 2018. The studentmonitor into IPD was responsible for accompanying the teacher in the theoretical and practical field. The theoretical moments happened in the classroom, by checking the content taught, the organization and the planning of revisions and support materials for academics. The practice moment itself, was divided into IPD in primary care and hospital IPD, in which the student-monitor, along with the instructor, acted on the orientation and execution of activities, which provided integration into teaching activities, overcoming the professional daily's challenges, giving him experiences of consolidation for his formation. Conclusion: The monitoring program broadens spaces of learning and contribute for a critical professional formation, reflexive and aligned with the superior magisterium.
\end{abstract}

Key words: Monitoring, Teaching, Learning.

${ }^{1}$ Universidade do Estado do Pará (UEPA), Belém-Pará. * E-mail: carla.costa09@hotmail.com

SUBMETIDO EM: 4/2019

ACEITO EM: 5/2019

PUBLICADO EM: 6/2019

REAS/EJCH | Vol. 11 (11) | e579 | DOI: https://doi.org/10.25248/reas.e579.2019 Página 1 de 5 


\section{RESUMEN}

Objetivo: relatar la experiencia en el proceso de enseñanza-aprendizaje de la monitoría académica del componente curricular de enfermería en enfermedades infecciosas y parasitarias (EIP). Relato de experiencia: se trata de un estudio descriptivo, con un enfoque cualitativo, del tipo de experiencia, basado en la experiencia del seguimiento académico del componente curricular de la EIP en la Universidade do Estado do Pará, durante el período comprendido entre septiembre y diciembre de 2018. El alumno-monitor en EIP fue el responsable de acompañar al profesor en el campo teórico y práctico. Los momentos teóricos pasaran en la clase, verificando el contenido en ministrados, en la organización y planificación de revisiones y materiales de apoyo para los académicos. El momento de la práctica se subdividió en EIP en la atención básica y la EIP hospitalar, en lo cual el alumno-monitor, junto con el profesor, actuó en la orientación y ejecución de las actividades, lo que le proporcionó la integración en las actividades docentes, superando los desafíos de la vida cotidiana profesional, proporcionando oportunidades de consolidación para su formación. Consideraciones finales: el programa de monitoría amplía los espacios de aprendizaje y contribuye a una formación profesional crítica, reflexiva y alineada con el magisterio superior.

Palabras clave: Monitoría, Enseñanza, Aprendizaje.

\section{INTRODUÇÃO}

Durante o exercício da profissão de Enfermagem nos deparamos com um mercado que exige profissionais com competências técnicas e científicas pautadas em princípios éticos e humanísticos (FRISON LMB, 2016). Nesse cenário, a educação superior tem papel relevante na formação de futuros enfermeiros, através de um ensino crítico e construtivo, proporcionando a troca de saberes e experiências, mediando a construção de cidadãos com senso político, crítico e reflexivo (MAGALHÃES LD, et al., 2014).

Para atingir as demandas atuais, as Instituições de Ensino Superior (IES) têm desenvolvido programas que visam o aperfeiçoamento do processo ensino-aprendizagem aos alunos de graduação. Dentre esses programas, destaca-se a monitoria acadêmica, considerada como uma estratégia de ensino, que tem o papel de fortalecer o ensino de graduação, por meio da atuação de monitores em práticas e experiências pedagógicas, em componentes curriculares que possibilitam o vínculo no campo teórico-prático. Propõe-se, igualmente, propiciar ao graduando condutas emancipadoras perante o conhecimento, incumbindo-se com maior responsabilidade, o compromisso de investir em sua formação (BACKES DS, et al., 2012).

A monitoria acadêmica foi regulamentada pela Lei oㅜ 5.540/1968 na qual fixou as normas de organização e funcionamento do ensino superior em conjunto com a escola média. Em 1996, esta foi reiterada pela Lei no 9.394/96, estabelecendo-se as diretrizes e bases da educação nacional e inserindo as atividades de ensino e pesquisa, compreendidas aqui como primordiais no desenvolvimento humano e acadêmico para a formação superior de estudantes a nível nacional (ANDRADE EGR, et al., 2018).

Segundo a Resolução № 2808 de 18 de Março de 2015, do Conselho Superior Universitário (CONSUN), que fixa as normas complementares para a execução do programa de monitoria na conjuntura da Universidade do Estado do Pará (UEPA), a monitoria tem como ponto-chave o engajamento do aluno de graduação na vida acadêmica, a partir do desenvolvimento de atividades supervisionadas ao ensino, da mesma forma, contribuir profissionalmente na formação acadêmica do discente-monitor, por meio do aperfeiçoamento teórico e/ou prático no componente curricular de seu interesse e no aprimoramento de habilidades de docência no discente-monitor.

Como evidenciado por Crisóstomo LCS, et al. (2018) o ambiente da sala de aula trás dificuldades aos estudantes, como sobrecarga de assuntos, confusão na compreensão de determinados conteúdos relacionados à disciplina, que se tornam um empecilho e são considerados fatores determinantes à desistência dos alunos. Esse quadro gera, então, indicadores negativos para a instituição, e como forma de 
se superar tal realidade a monitoria também tem o objetivo de motivação e incentivo para um melhor rendimento acadêmico dos discentes.

Ainda que o protagonista no processo de ensino-aprendizagem seja o aluno, o docente também possui um papel primordial no que tange proporcionar um bom arcabouço para a formação acadêmica, assim como os monitores que bem formados e entendedores do seu papel no contexto de ensino-aprendizagem, influenciam diretamente neste processo (ABREU TO, et al., 2014).

Santos GM e Batista SHSS (2015), revelam que a monitoria é tida como um meio de aprofundar os próprios conhecimentos adquiridos na vida acadêmica, ajudando outros alunos na absorção de conteúdos explanados, como também, aproxima o discente-monitor da docência, seja pela associação com os discentes e docentes ou pelo modo de conduzir atividades referentes ao componente.

A monitoria tende a ser considerada como uma atividade que requer a capacidade do discente-monitor de atuar como um intermediador da aprendizagem dos discentes em combinação com os docentes. Nessa perspectiva, o monitor atua como orientador e organizador das propostas de ensino na formação universitária (CARVALHO IS, et al., 2012).

Ademais, a monitoria proporciona ferramentas que contribuem no processo ensino-aprendizagem, tanto para o aprendizado dos discentes como também do discente-monitor, proporcionando aos acadêmicos subsídios adaptativos às demandas atuais, renovando variedades no processo de aprendizagem, como também atuando na problematização em saúde trazendo resolubilidade nos diferentes campos de atuação (SANTOS GM e BATISTA SHSS, 2015).

Por conseguinte, Fernandes $\mathrm{J}$ et al. (2016) complementa que a monitoria acadêmica possibilita experimentar situações que Ihe proporcionam amadurecimento, quando em contato com as distintas áreas e problemas que futuramente serão enfrentados. Além disso, propicia o rompimento de barreiras como comunicação, desinteresse, falta de dedicação entre discentes, monitores e docentes.

A monitoria constitui, um principal elemento facilitador para o desenvolvimento de conhecimentos, permitindo a correlação eficiente entre teoria e prática, criando um espaço favorável onde o discente pode criar, questionar, praticar e revisar conteúdos ministrados intra e extramuros da instituição de ensino, com menos de receio e maior nível de segurança e participação. E acima de tudo, possibilita a incorporação do discente-monitor em atividades docentes, aprimorando e superando os desafios do cotidiano profissional, proporcionando-Ihe experiências de consolidação de sua formação acadêmica (JÚNIOR AFC, et al., 2017).

O despertar por esse tema surgiu em decorrência do exercício da monitoria do componente curricular "Enfermagem em Doenças Infectocontagiosas e Parasitárias" (DIP), na graduação em Enfermagem da UEPA. Desta forma, o objetivo desse estudo é relatar a experiência no processo ensino-aprendizagem da monitoria acadêmica do componente curricular Enfermagem em DIP.

\section{RELATO DA EXPERIÊNCIA}

Trata-se de um estudo descritivo, de abordagem qualitativa, do tipo relato de experiência, baseado na vivência da monitoria acadêmica, durante o período de setembro a dezembro de 2018, após a aprovação no processo seletivo de no 52 - 2018, para o componente curricular Enfermagem em DIP. O referido componente integra a grade curricular do $8^{\circ}$ semestre do curso de Graduação em Enfermagem da UEPA, inserido tanto no Departamento de Enfermagem Comunitária (DENC) como também no Departamento de Enfermagem Hospitalar (DENH), tendo carga horária de 180 horas, subdivididas em 60 horas voltadas para o campo teórico e 120 horas para o campo prático.

De acordo com a resolução oㅡ 2808/15 do CONSUN, a monitoria tem durabilidade de 6 meses, podendo ser prorrogada por igual período, possuindo carga horária semanal de 15 horas, totalizando 60 horas mensais. Desta forma, no que tange ao componente curricular, a carga horária semanal é distribuída em 4 horas semanais para participação em aulas teóricas e 11 horas para o campo de prática.

REAS/EJCH | Vol. 11 (11) | e579 | DOI: https://doi.org/10.25248/reas.e579.2019 Página 3 de $\mathbf{5}$ 
A disciplina tem como objetivo, dar subsídios quanto à promoção, prevenção e reabilitação da saúde, diagnóstico e tratamento de doenças, aplicando ações de controle em nível epidemiológico, ambiental, político e assistencial para o manejo das doenças endêmicas mais prevalentes na região Amazônica. Os conteúdos ministrados no componente curricular DIP, são: Atuação do enfermeiro na prevenção e controle da hanseníase, tuberculose, acidentes ofídicos, raiva, doença de chagas, HIV e AIDS, malária, dengue, zika, chikungunya, febre amarela, leptospirose, febre tifoide, dentre outras doenças infecciosas e parasitárias que são prevalentes no contexto Amazônico.

Tal componente desenvolve suas atividades didático-pedagógicas em três ambientes, nos quais o alunomonitor deve participar: A sala de aula, para o acompanhamento das aulas teóricas, cujos conteúdos são ministrados em dois dias da semana; em um Centro Saúde Escola (CSE) para a atuação prática na atenção básica; e em um hospital de referência regional em Pneumologia, Infectologia, Endocrinologia, Diabetes, e referência nacional em AIDS para a atuação prática do componente na atenção hospitalar.

Durante o período teórico da monitoria acadêmica, o aluno-monitor era resignado em acompanhar as aulas ministradas pelos docentes, com o caráter participativo, tendo como objetivo, assimilar os conteúdos, para em seguida organizar as aulas de revisão e elaborar exercícios de fixação para os acadêmicos, com o intuito de amenizar ou suprir as dificuldades e dúvidas do componente curricular exibidos pelos mesmos.

Dessa forma, evidencia-se como primeiro desafio da monitoria, ministrar revisões, organizando e planejando materiais de cunho didático para os acadêmicos, assim como aprofundar a leitura e conhecimento nos conteúdos do componente curricular estudados para a posterior exposição da explicação e retirada de dúvidas, sendo também necessário o conhecimento de metodologias de ensino-aprendizado que facilitassem a compreensão e assimilação significativa dos estudantes sobre os conteúdos que estavam sendo revisados.

No CSE, houve um acompanhamento em conjunto com os discentes e a docente no campo de prática de DIP na Atenção Básica, nos meses de setembro e outubro. Foram realizadas consultas de enfermagem aos usuários em tratamento de Tuberculose e Hanseníase, atendimentos no consultório destinado ao Centro de Testagem e Aconselhamento (CTA) com testagens rápidas de HIV, Hepatites B e C e Sífilis. Na sala de Imunização, pôde-se observar todas as etapas do processo do acondicionamento dos imunobiológicos e da vacinação.

Antes da realização das consultas de enfermagem, com usuários em tratamento de tuberculose e hanseníase, o aluno-monitor realizava simulações de consultas de enfermagem com os discentes, com o propósito de fixar as condutas para uma consulta adequada, onde o monitor atuava na condição de usuário e os discentes nas condições de enfermeiros. Na simulação realística, o monitor descrevia o caso clínico proposto e os discentes deveriam apresentar as condutas necessárias para o manejo de cada caso. Ao final desse momento, o discente-monitor possuía a tarefa de destacar e explanar os pontos positivos e negativos na consulta de enfermagem efetuada por cada aluno. Após a simulação e da elucidação dos pontos avaliados, os discentes realizavam as consultas de enfermagem propriamente ditas, sempre sob supervisão do docente em conjunto com o monitor.

Na sala do CTA, era estipulado ao monitor as explicações detalhadas sobre o funcionamento e o fluxo da sala, adequação na entrevista com os usuários, o preenchimento adequado dos formulários, o processo de notificação e as etapas a serem seguidas na coleta do material para a testagem rápida, sempre atuando com o docente responsável. Nessa ocasião, o desafio do processo ensino-aprendizagem estava na interação com os acadêmicos e explicação apropriada de todos os processos condizentes para a atuação em um centro de testagem, tal integração era sempre intermediada pelo docente responsável.

$\mathrm{Na}$ Imunização, o monitor acompanhava os alunos, tendo o papel de explicar o processo de armazenamento dos imunobiológicos na câmara refrigerada, geladeira e na caixa térmica, as características da sala, manejo para o plano de contingência, como se sucede o aprazamento do cartão de vacinação e as características dos imunobiológicos. Com isso, pode-se perceber, que o aluno-monitor atuante em DIP, deve ter o conhecimento aprofundado sobre o processo de imunização, ter a capacidade teórica de explanar o 
conteúdo de modo que o discente possa entender. Desta forma, o aqui apresentado estava em relembrar o conteúdo já estudado anteriormente e explicação efetiva.

As atividades desempenhadas no período final de cada prática, sucederam-se com a elaboração e execução de ações de educação em saúde com os usuários do CSE, e os temas deveriam ser condizentes com os ministrados na disciplina. Com isso, o aluno-monitor em DIP vivenciou com os discentes a construção e organização de um planejamento estratégico, que deveria conter uma fundamentação teórica, objetivos, metodologia, os materiais a serem utilizados, a durabilidade da ação, a dinâmica a ser desenvolvida, a função de cada integrante na ação e os possíveis resultados esperados. Bem como, esse planejamento deveria ser seguido fidedignamente no momento da ação, sob supervisão do docente.

Durante os meses de novembro e dezembro as práticas ocorreram em um hospital referência de modo a se efetivar o processo de ensino sobre a enfermagem na atenção terciária. Desempenhou-se atividades como a Sistematização da Assistência de Enfermagem (SAE), exame físico, curativos e sondagens.

No campo de prática hospitalar, foram executados o acompanhamento dos alunos em suas atividades práticas, o monitor atuava no auxilio das perguntas adequadas a serem feitas para o usuário, na condução do exame físico, no elucidação das dúvidas acerca do processo de curativo e na passagem de sonda, na construção da evolução de enfermagem de cada usuário nos prontuários, auxiliando na ordenação da evolução e nos termos técnicos a serem utilizados na escrita. O desafio vivenciado na monitoria durante o campo de prática hospitalar de DIP foi a construção, em conjunto com os discentes, de um estudo de caso encontrado durante a prática. Tal estudo englobou os dados epidemiológicos, sinais e sintomas, diagnóstico e a SAE, socializados no último dia de prática do componente o docente.

\section{CONSIDERAÇÕES FINAIS}

Diante do exposto, percebe-se que a atuação do monitor no campo teórico e prático, seja no âmbito da Atenção Primária ou hospitalar, traz benefícios para a tríade envolvida no processo da monitoria acadêmica (docente, discentes acompanhados e aluno-monitor). Dessa forma, reiteramos a importância de se fortalecer cada vez mais o Programa de Monitoria, pois este proporciona um melhor rendimento dos acadêmicos na disciplina proposta, na consolidação indutiva de uma formação profissional crítico e reflexivo e coerente com a realidade, com aprimoramento de habilidades e conhecimento, como também, tornando-se ferramenta estimuladora para quem deseja atuar no magistério superior.

\section{REFERÊNCIAS}

1. ABREU TO, et al. A monitoria acadêmica na percepção dos graduandos de enfermagem. Rev Enferm UERJ, 2014; 22(4): 50712.

2. ANDRADE EGR, et al. Contribuição da monitoria acadêmica para o processo ensino-aprendizagem na graduação em enfermagem. Rev Bras Enferm. 2018; 71(4): 1690-1698.

3. BACKES DS, et al. Vivência teórico-prática inovadora no ensino de enfermagem. Esc Anna Nery Rev Enferm, 2012; 16(3): 597-602.

4. CARVALHO IS, et al. Monitoria em semiologia e semiotecnica para a enfermagem: um relato de experiência. Rev Enferm UFSM. 2012; 2(2): 464-471.

5. CRISÓSTOMO LCS, et al. Contribuição do Programa de Monitoria Acadêmica (PROMAC/UECA) na formação do futuro professor de química da região do Vale de Jaquaribe-CE. Revista educacional interdisciplinar, 2018; 7(1): 1-10.

6. FERNANDES J, et al. Influência da monitoria acadêmica no processo de ensino aprendizagem da psicologia. Clínica e Cultura, 2016; 2(1); 36-43.

7. FRISON LMB. Monitoria: uma modalidade de ensino que potencializa a aprendizagem colaborativa e autorregulada. Revista Pro-Posições, 2016; 27(1): 133-153.

8. JÚNIOR AFC, et al. Monitoria acadêmica e metodologia da problematização: relato de experiência. Revista Ciência em Extensão, 2017; 13(3): 136-145.

9. MAGALHÃES LD, et al. A monitoria acadêmica da disciplina de cuidados críticos para a enfermagem: relato de experiência. Revista da Universidade Vale do Rio Verde, 2014; 12(2): 556-565.

10. SANTOS GM, BATISTA SHSS. Monitoria acadêmica na formação em/para a saúde: desafios e possibilidades no âmbito de um currículo interprofissional em saúde. ABCS Health Sci, 2015; 40(3): 203-207.

11. UNIVERSIDADE DO ESTADO DO PARÁ. Conselho Universitário. Resolução no 2.808/15 - CONSUN, 18 de março de 2015; 2015. 15 p. Fixa normas complementares para execução do programa de monitoria no âmbito da Universidade do Estado do Pará. 